\title{
Demographics and Pharmacotherapy in Neurocysticercosis: A hospital Based Study
}

\author{
Resha Shrestha ${ }^{1}$, Pranaya Shrestha ${ }^{1}$, Pravesh Rajbhandari ${ }^{1}$, Samir Acharya ${ }^{1}$, Avinash Chandra ${ }^{1}$, Sujat \\ Dahal $^{1}$, Joshan Neupane ${ }^{1}$, Pritam Gurung ${ }^{1}$, Basant Pant ${ }^{1}$ \\ ${ }^{1}$ Department of Neurosurgery, Annapurna Neurological Institute \& Allied Sciences, Maitighar, Kathmandu, Nepal
}

\section{CORRESPONDENCE}

Dr. Resha Shrestha,

Department of Neurosurgery, Annapurna Neurological Institute \& Allied Sciences,Maitighar, Kathmandu,Nepal

Email: reskums@hotmail.com

\section{ARTICLE INFO}

Article History

Submitted: 26 November, 2020

Accepted: 21 December, 2020

Published: 8 February, 2021

Source of support: None Conflict of Interest: None

Copyright : (CThe Author(S) 2020 This is an open access article under the Creative Common Attribution license CC-BY 4.0

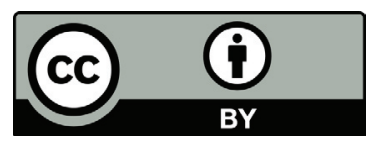

\begin{abstract}
Introduction: Neurocysticercosis (NCC) is one of the commonest preventable cause of seizure. It is due to development of the larval form of Taenia solium. This disease is endemic in south east Asia including Nepal and has been considered as one of the neglected tropical disease.
\end{abstract}

Methods: All the patients who came to Neurosurgical outpatient department (OPD) of Annapurna Neurological Institute and Allied Sciences (ANIAS) with the diagnosis of NCC with seizure during the study time frame were included in the study. The time frame of the study was January 1st 2017 till December 31st 2018.

Results: There were 167 total cases of which 108 (64.7\%) were male and 59 (35.3\%) were female. Mean age was 27.9 years (SD 13.1 years, range 1 year to 66 years). Frequency of patients were in the age group 20-30 years followed by $10-20$ years. In terms of number of lesions, $86 \%$ of the patients had single lesion while $14 \%$ had multiple lesionsMaximum number of cases had focal seizure with secondary generalization (46\%). This was followed by focal seizure (28\%). Most common antiepileptics was carbamazepine (51.5\%). Mean duration of antiepileptics was 2.5 years (SD 1.47, Range 9 months to 6 years) and recurrence was noted in $7.78 \%$ of cases.

Conclusion: NCC affects the productive age group with higher proportion being male patient. Single lesion is more prevalent. Proper measures for treatment and prevention of neurocysticercosis is essential and can lead to better control of this condition.

Keywords: Demographics; Neurocysticercosis (NCC); Pharmacotherapy.

\section{INTRODUCTION}

Neurocysticercosis (NCC) is one of the commonest preventable cause of seizure. This is caused by ingestion of tapeworm eggs. ${ }^{1} \mathrm{NCC}$ is due to development of the larval form of Taenia solium. It should be noted that NCC is only acquired by ingestion of eggs (fecal-oral route) and not due to ingestion of cysticerci in undercooked pork which causes intestinal taeniasis. ${ }^{2}$ Though humans are the definitive hosts for Tinea solium, cysticercosis results from human acting as accidental intermediate hosts for the parasite. ${ }^{1}$

This disease is endemic in south east Asia including Nepal and has been considered as one of the "neglected tropical disease". ${ }^{3,4}$ It is estimated that 50 million people worldwide has NCC with approximately 50,000 deaths each year relating to NCC. NCC is responsible for more than half cases of late onset epilepsy in developing countries. ${ }^{5,6}$ The prevalence of NCC in Nepal is 0.002-0.1\% in general public. Still existing open defecation, backyard pig raising might be a reason for NCC to be endemic in Nepal. ${ }^{7,8}$ Studies in Nepal have shown that up to 7.3 per 1,000 population may suffer from epilepsy with about $50 \%$ of the cases attributed to NCC. ${ }^{9}$

There is paucity of recent NCC data in Nepal. Knowing the details of outpatient data of NCC patients help to know the disease burden in our context. Also knowing the details of involvement of NCC in seizure cases help to know the course and duration of treatment of it.

\section{METHODS}

All the patients who came to Neurosurgical outpatient department (OPD) of Annapurna Neurological Institute and Allied Sciences (ANIAS) with the diagnosis of NCC with seizure during the study time frame were included in the study. The time frame of the study was January 1st 2017 till December 31st 2018. Data on demographic 
parameters, number of lesions and pharmacotherapy were entered. Also data on need of admission, surgical procedures were also entered. Database was created in microsoft access and was then transferred to SPSS 18.0 for statistical analysis. Scalar variables were expressed in terms of mean, standard deviation and range. Nominal variables were expressed in terms of frequency and percentage. The study was conducted after obtaining ethical clearance from the institutional review committee.

\section{RESULTS}

There were 167 total cases of which 108 (64.7\%) were male and 59 (35.3\%) were female. Mean age was 27.9 years (SD 13.1 years, range 1 year to 66 years). Figure 1 shows the number of patients in different age groups.It was found that the maximum frequency of patients were in the age group $20-30$ years followed by $10-20$ years.

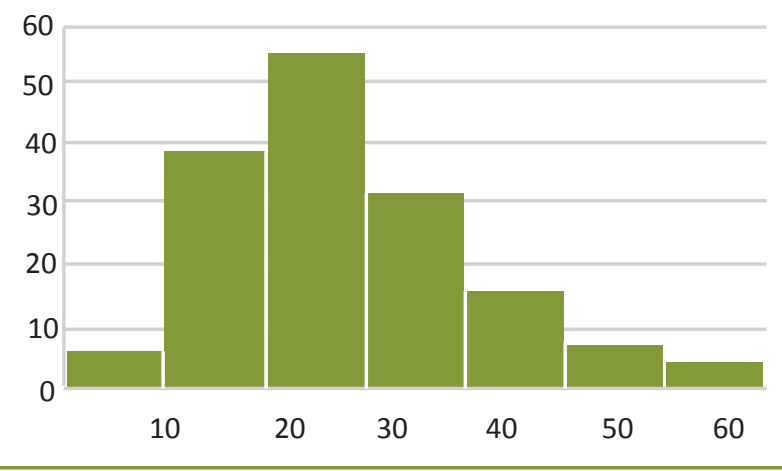

Figure 1: Number of patients in different age groups.

Figure 2 shows the number of patients from different development regions of Nepal. It was found that highest number of patients were from the capital followed by western and eastern Nepal. Although provincial system is present in Nepal, during the time of this study, development regions was present.

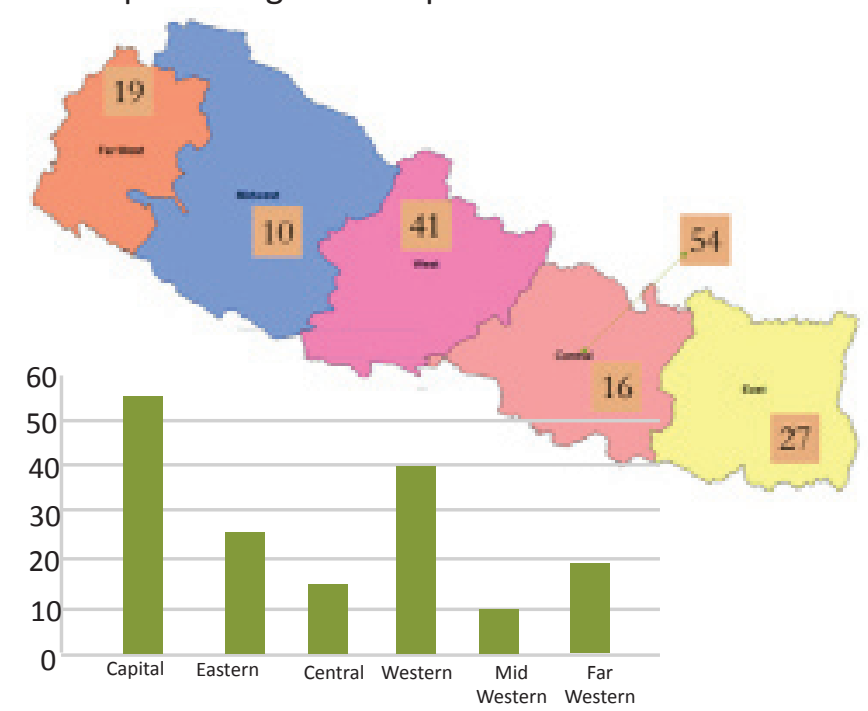

Figure 2: Number of patients from different developmental regions and capital of Nepal
In terms of number of lesions, $86 \%$ of the patients had single lesion while $14 \%$ had multiple lesions. In $90.4 \%$ imaging modality done was Computed Tomography (CT) which was both plain and enhanced CT. In 9.6\% imaging modality was Magnetic Resonance Imaging (MRI). Figure 3 shows typical finding of NCC lesion in CT and MRI respectively.

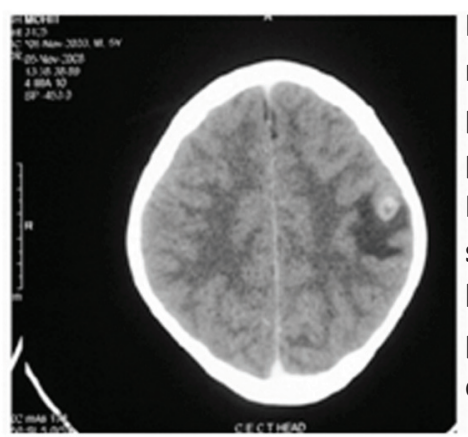

Figure 3 a: CT scan showing ring enhancing lesion with perilesional edema in left parietal region.

Figure 3b: MRI brain showing ring enhancing lesion with two scolex with perilesional edema in left occipital region.

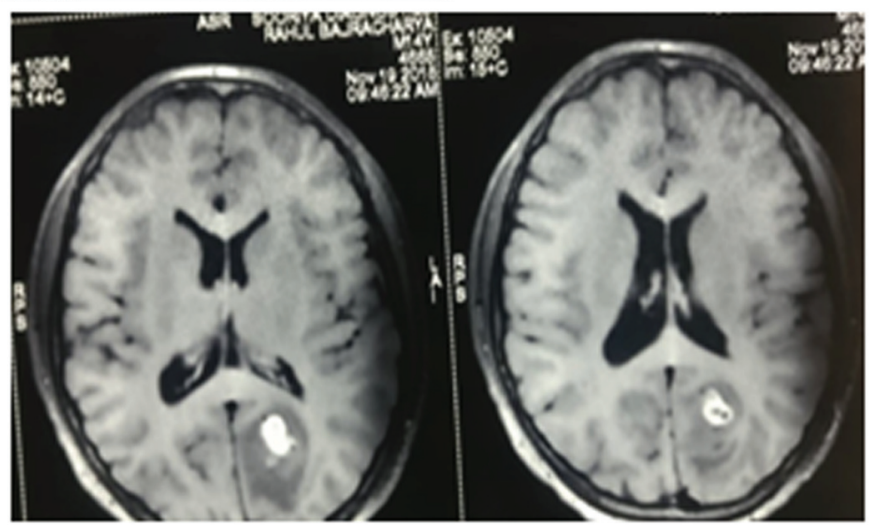

Figure 3: Typical finding of NCC lesion in CT and MRI respectively.

percentage of cases in different seizure types. Maximum number of cases had focal seizure with secondary generalization (46\%). This was followed by focal seizure (28\%).

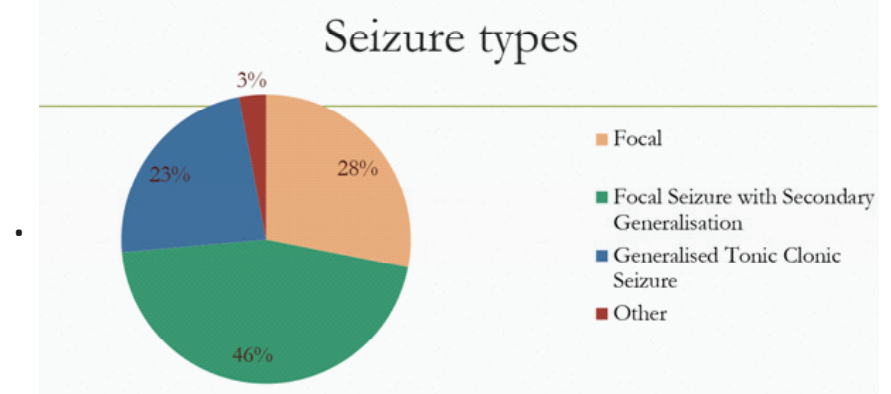

Figure 4: Percentage of cases in different seizure types

Figure 5 shows percentage of cases in different treatment duration. Most number of cases had more than 2 years of treatment $(74.85 \%)$ followed by 9 months of treatment (17.36\%). In 7.78\% of cases there was history or recurrence. Most common antiepileptics was carbamazepine (51.5\%) followed by valproic acid (26.3\%) and Levercetam. In patients who had received carbamazepine, $6 \%$ had episode 


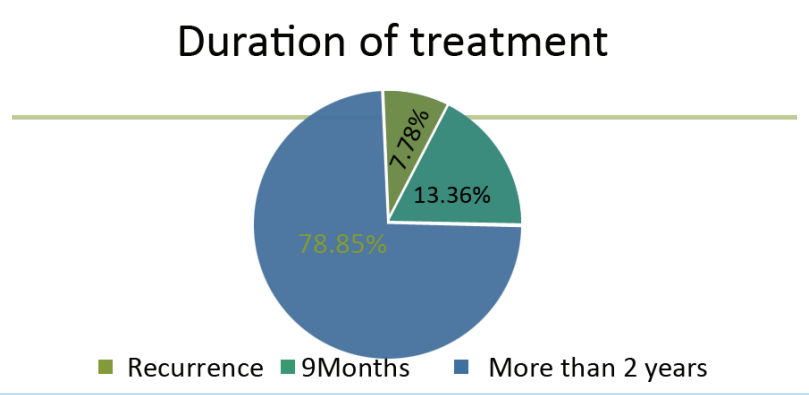

Figure 5: Percentage of cases in different treatment duration

of Stephen Johnson Syndrome. Mean duration of antiepileptics was 2.5 years (SD 1.47, Range 9 months to 6 years). In our center, total number of outpatient visits in Neurosurgical OPD in 2017 was 18,545 and that in 2018 was 19,663 . The total number of seizure patients in OPD in 2017 was 1060 and that in 2018 was 634. Thus percentage of seizure cases among outpatient cases in Neurosurgical OPD in 2017 was $5.7 \%$ while that in 2018 was $3.2 \%$. The overall percentage of cases of seizure in the year 2017 and 2018 combined was $4.4 \%$. NCC as cause of seizure was present in $9.8 \%$. During the same time there were total 59 admission for NCC. In regards to surgical procedures required for NCC, lesionectomy was done in two patients who had intractable seizure due to the scar in the brain.

\section{DISCUSSION}

World Health Organization has classified NCC as the most important parasitic neurologic disease.$^{10}$ In a study to know the burden of parasitic zoonosis in Nepal, it was found that NCC was associated with highest annual burden. ${ }^{11}$ Although prevalence of NCC hasn't been studied well in our country, studies have found that prevalence of taeniasis in five different districts of Nepal ranged from $11 \%$ to $43 \%$. ${ }^{12}$ These studies have found that the prevalence was more in the western part of Nepal and risk of cysticerciwas more in Banke district. We had more number of patients from the central region of Nepal which might be due to the proximity of hospital in the central region.Study by Heap in 1998 reported that NCC is a common cause of epilepsy in Nepalese soldiers in Hongkong. ${ }^{13} \mathrm{NCC}$ is a single most common cause of epilepsy in Nepal. ${ }^{14}$ In a study done at Bir hospital, Kathmandu, Out of 131 seizure patients admitted, 21 patients were diagnosed with NCC (16\%). ${ }^{15}$ In the study, mean age of the patients was 33.95 years, with $71.4 \%$ male patients and $28.6 \%$ female patients. The proportion of male patient was also high compared to female patients in our study too.

In another study done at Dharan, of the 114 in-patient cases of neurocysticercosis, $63 \%$ patients were male and $42 \%$ were in age group <20years. ${ }^{16}$ Proportion of male patients in this study is very similar to our series. Mean age in 62 histopathology proven case of NCC patients was 21 years which was slightly lower than our finding of 27.9 years. ${ }^{17}$ Many clinical experience of NCC reported from Nepal have found different presentation and treatment responses. ${ }^{18,19}$

Commonly followed classification based on imaging finding differentiates living (Stage I), dying (Stage II) and dead status (Stage III) of the cyst with finding of hypodense cyst, ring or disc enhancing lesion, hyperdense lesion without enhancement respectively. This has been further classified as presence or absence of edema especially in stage II and III. ${ }^{8} \mathrm{~A}$ case series conducted in Nepal have reported three cases of intraventricular Taenia solium causing neurological symptoms. ${ }^{20}$

The commonest Antiepileptic drugs prescribed in our patients were carbamazepine as focal seizure and focal seizure with secondary generalization was the commonest presentation. The duration of treatment was 9 months if the lesion disappeared in the follow up scan in three months period. However in $74.8 \%$ of our patient we gave AED for two years which suggested that the lesion did not disappear in the follow up Scan and we tapered AED once the EEG was normal. We had $7.74 \%$ of recurrent seizure. It has been stated thatpresence of calcific residue on the CT scan, occurrence of breakthrough seizures, and occurrence of more than two seizures were associated with a higher risk of recurrence of seizures. ${ }^{21}$ The recurrence rate was $15 \%$ in this study. We did not prescribe antiparaite drug (albendazole)therapy in single lesion as there is no need of antiparasite drugs in single lesion and if it it is a calified lesion then the cyst is already dead..$^{22}$ In terms of number of lesions in a patient, our study found more prevalence of single lesion. Similar finding was also seen in India where as multiple lesion was found in Latin America. ${ }^{23}$

In current study, most common seizure was focal seizure secondary generalized followed by generalized tonicclonic seizure. In earlier retrospective study of 250 patients at our institute who presented with seizures; it was found that $47.1 \%$ of the patients had Generalized Tonic ClonicSeizure. This was followed by focal seizure secondary generalized. ${ }^{8}$ NCC is considered an eradicable disease by public health experts if appropriate widespread preventive measures are followed. ${ }^{24}$

\section{CONCLUSION}

NCC affects the productive age group with higher proportion being male patient. Single lesion is more prevalent. Proper measures for treatment and prevention of neurocysticercosis is essential and can lead to better control of this condition. 


\section{REFERENCES}

1. Gripper LB, Welburn SC. Neurocysticercosis infection and disease-A review. Acta Trop. 2017;166: 218-224.

2. Del Brutto OH. Neurocysticercosis. Continuum . 2012;18: 1392-1416.

3. Dorny P, Praet N, Deckers N, Gabriel S. Emerging foodborne parasites. Veterinary Parasitology. 2009;163:196206.

4. Fabiani S, Bruschi F. Neurocysticercosis in Europe: Still a public health concern not only for imported cases. Acta Tropica. 2013;128:18-26.

5. Tellez-Zenteno JF, Hernandez-Ronquillo L. Epidemiology of neurocysticercosis and epilepsy, is everything described? Epilepsy Behav. 2017;76: 146-150.

6. Joshi DD, Bista PR, Ito A, Yamasaki H. Present Situation of Porcine Taeniasis and Human Cysticercosis in Nepal. South Asian J Trop Med Public Health. 2007;38:144-50.

7. Devleesschauwer B, Aryal A, Joshi DD, Rijal S, Sherchand JB, Praet N, et al. Epidemiology of Taenia solium in Nepal: is it influenced by the social characteristics of the population and the presence of Taenia asiatica? Trop Med Int Health. 2012;17: 1019-22.

8. Joshi B, Pant B, Rajbhandari P, Ajanga RP, Malla R, Manandhar KD, Sreerama L. The Epidemiology and Health Burden of Neurocysticercosis in Nepal. International Journal of Trop Disease\& Health. 2014;4: 204-23.

9. Rajbhandari KC. Epilepsy in Nepal. Canadian Journal of Neurological Sciences / Journal Canadien des Sciences Neurologiques. 2004;31:257-60.

10. Scheel CM, Gonzalez AE, Garcia HH, Tsang VCW, Hancock $\mathrm{K}$, Gilman RH, et al. Serodiagnosis of Neurocysticercosis of Assay Formats. The American Journal of Tropical Medicine and Hygiene. 2005:73: 771-6.

11. Devleesschauwer B, Ale A, Torgerson P, Praet N, Maertens de Noordhout C, Pandey BD, et al. The burden of parasitic zoonoses in Nepal: a systematic review. PLoSNegl Trop Dis. $2014 ; 8$.

12. Joshi DD, Maharjan M, Johnson MV, Willingham AL, Gaihr Y, Sharma M. Taeniasis/cysticercosis situation in Nepal. Southeast Asian J Trop Med Public Health 2004; 35 (1):252-7.

13. Del Brutto OH, Sotelo J, Raman GC. Neurocysticercosis: A Clinical handbook. Swets\&Zeitlinger B. V. 1998.

14. Pant B, Lin N. An epidemiological study of An epidemiological study of neurocysticercosis in Nepal. Proceeding of the Society of Internal Medicine of Nepal, 2002; 17.

15. Ojha R, Shah D, Shrestha A, Koirala S, Dahal A, Adhikari K, et al. Neurocysticercosis in Nepal: a retrospective clinical analysis. Neuroimmunology and Neuroinflammation. 2015;2:167-170.

16. Sah RB, Subedi L, Pandey N, Sah BP, Jha S, Shah U, et al. The epidemiology and health burden of neurocysticercosis in tertiary hospital of Nepal. Journal of Chitwan Medical College. 2014:4;13-16.

17. Amatya BM, Kimula Y. Cysticercosis in Nepal: a histopathologic study of sixty-two cases. Am J Surg Pathol. 1999;23: 1276-1279.

18. Shrestha BM. Childhood Neurocysticercosis: Clinicoradiological profile \& outcome. J Nepal Paediatr. Soc. 2008;28(1): 14-16.

19. Panta B. Neurocysticecosis; a major cause of seizure in Nepal. Proceedings of present situation challenges in treatment and elimination of Taeniasis/cysticercosis in Nepal,edited by Joshi DD, Sharma M, Rana S. National Zoonoses \& Food Hygeine Research Centre, Kathmandu:2006:55-73.

20. Pant B, Devleesschauwer B, Shrestha P, Shrestha I, Praet N, Dorny P. Intraventricular Taenia solium neurocysticercosis: a report of three cases. JNMA J Nepal Med Assoc. 2011;51: 192-195.

21. Rajshekhar V, Jeyaseelan L. Seizure outcome in patients with a solitary cerebral cysticercus granuloma.Neurology. 2004;62(12):2236.

22. García HH, Evans CA, Nash TE, et al. Current consensus guidelines for treatment of neurocysticercosis. Clin Microbiol Rev 2002; 15:747.

23. Bobes RJ, Fragoso G, Fleury A, García-Varela M, Sciutto E, Larralde $\mathrm{C}$, et al. Evolution, molecular epidemiology and perspectives on the research of taeniid parasites with special emphasis on Taenia solium. Infection, Genetics and Evolution. 2014;4:150-160.

24. Agrawal JP. Neurocysticercosis in Nepal and It's Global Perspective. Kathmandu University Medical Journal. 2012;9:1-2. 